\title{
Evanescent Kerr effect using an optical nanofiber in acetone
}

\author{
Gil Fanjoux*, Jacques Chrétien, Adrien Godet, Kien Phan-Huy, Jean-Charles Beugnot, \& Thibaut Sylvestre \\ FEMTO-ST Institute, Optics Department, Université Bourgogne Franche-Comté, CNRS, Besançon - France
}

\begin{abstract}
We report on a theoretical and experimental investigation of the optical Kerr effect in the evanescent field of silica nanofibers immersed in several highly nonlinear liquids such as ethanol, acetone and water and we further compare them with air cladding. We provide formula of the effective nonlinear coefficients including the contribution of the nanofiber silica core and of the evanescent field for varying nanofiber diameter and for different surrounding media.
\end{abstract}

\section{Introduction}

Optical nanofibers are ultra-thin tapered optical fibers that feature unique optical properties, including a strong field confinement in the core, enhanced nonlinear effects, and a wide evanescent field that can interact with the surrounding medium and can therefore modify or detect chemical elements [1]. Among third-order nonlinear effects, stimulated Raman scattering (SRS) has recently been evidenced in the evanescent field of tapered silica nanofibers dipped in nonlinear liquids such as ethanol and toluene [2]. It has been shown in particular that the evanescent Raman effect in the highly nonlinear liquid is stronger than the standard Raman effect in the silica nanofiber itself. In this paper, we theoretically demonstrate that the evanescent Kerr effect can also be stronger than that in the silica taper. We further report the experimental observation of the evanescent Kerr effect through measurements of the stimulated Raman-Kerr scattering in a silica nanofiber immersed in acetone. The evanescent Kerr effect is shown to give rise to a strong asymmetric spectral broadening of the first Raman order generated in the nonlinear liquid.

\section{Theory and experiments}

The optical Kerr effect in a nonlinear optical waveguide is usually defined using the following nonlinear parameter

$$
\gamma=\frac{2 \pi n_{2}}{\lambda A_{e f f}}
$$

where $\mathrm{n}_{2}$ is the nonlinear Kerr index of the nonlinear medium, $\mathrm{A}_{\mathrm{eff}}$ the effective area of the guided mode, and $\lambda$ the wavelength of the field propagating in the waveguide. For a tapered fiber with a waist close to the optical wavelength, the evanescent field of the fundamental mode interacting with the external environment becomes more important than that the electric field guided into the core. To include the evanescent field into the Kerr effect, we can write the effective nonlinear parameter $\gamma_{\text {eff }}$ as

$$
\gamma_{e f f}=\frac{2 \pi}{\lambda} \frac{\iint_{-\infty}^{+\infty} n_{2}(x, y) S_{Z}^{2} d x d y}{\left(\iint_{-\infty}^{+\infty} S_{Z}^{2} d x d y\right)^{2}}
$$

where $\mathrm{S}_{\mathrm{z}}$ is the Poynting vector component in the waveguide direction. This equation is an approximation as it does not assume the $z$ field component, which is no more negligible for very small diameter [3]. However, although the propagating modes of a waveguide are not fully transverse in strong guidance regime, the full vectorial formalism of $\gamma_{\mathrm{eff}}$ and the simplified expression of Eq. (2) gives the same results of $\gamma_{\text {eff }}$ for silica nanofiber in air even for small core diameter down $0.3 \mathrm{~nm}$ [3]. If now the core and cladding have different Kerr coefficients, the previous Eq. (2) is written as

$\gamma_{e f f}=\frac{2 \pi n_{2, c o}}{\lambda} \frac{\iint_{c o} S_{Z}^{2} d x d y}{\left(\iint_{-\infty}^{+\infty} S_{Z}^{2} d x d y\right)^{2}}+\frac{2 \pi n_{2, c l}}{\lambda} \frac{\iint_{c l} S_{Z}^{2} d x d y}{\left(\iint_{-\infty}^{+\infty} S_{Z}^{2} d x d y\right)^{2}}(3)$

with $n_{2, c o}$ and $n_{2, c l}$ the core and cladding Kerr coefficient, respectively, considered as uniform. Then, the first term of Eq. (3) corresponds to the contribution to the Kerr effect of the silica core, while the second one is the contribution of the cladding, i.e., $\gamma_{\mathrm{eff}}=\gamma_{\mathrm{co}}+\gamma_{\mathrm{cl}}$. To get the effective Kerr coefficient, we first computed the $S_{z}$ Poynting component of the fundamental mode using a finite element method. The studied system corresponds to a silica nanofiber with a various diameter, and surrounding by air or liquid. Parameters used are laser wavelength of $532 \mathrm{~nm}$, refractive index of 1.4606, 1.000, 1.3337, 1.3637 and 1.3614 for silica, air, water, ethanol and acetone, respectively, and the corresponding nonlinear Kerr indices $\mathrm{n}_{2}$ are 2.6, 3.10 $0^{-3}, 4.1,7.7$ and 24 $\left(\times 10^{-20} \mathrm{~m}^{2} / \mathrm{W}\right)$.

Figs. 1(a-d) show the nonlinear parameters of the silica core $\gamma_{\mathrm{co}}$ and of the cladding $\gamma_{\mathrm{cl}}$, the effective nonlinear coefficient $\gamma_{\text {eff }}$ and the evanescent Kerr rate, all as a function of the nanofiber diameter. Concerning the silica core, $\gamma_{c o}$, it first increases until reaching a maximum value for diameters close to those of the minimal effective area. This is because if the effective mode area is very weak, the field intensity and therefore the Kerr effect in the silica

\footnotetext{
Corresponding author: gil.fanjoux@,univ-fcomte.fr
} 

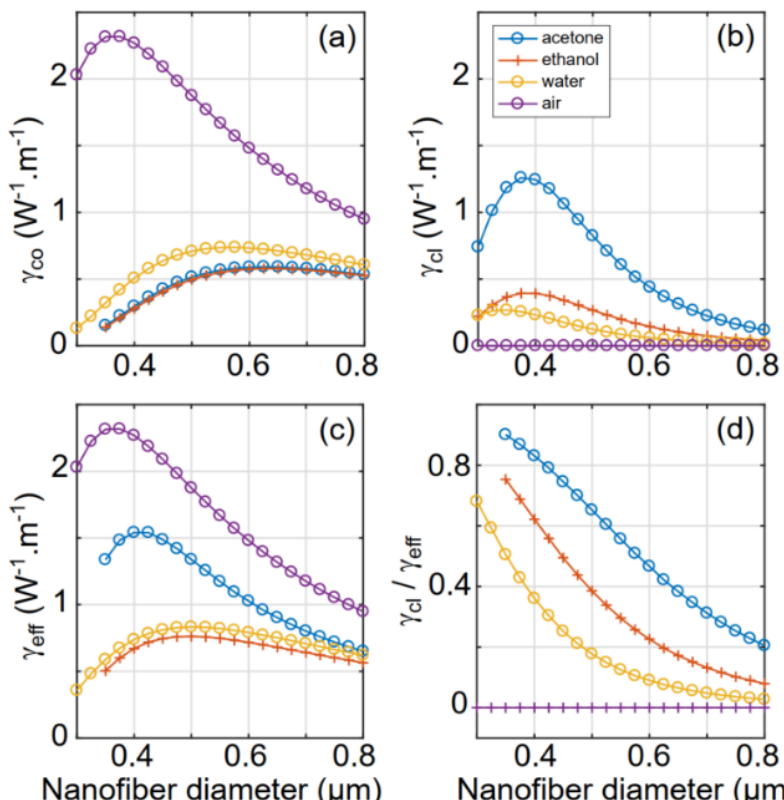

Nanofiber diameter $(\mu \mathrm{m})$

Figure 1. Evolution in function of the nanofiber diameter and for different surrounding media of (a) the nonlinear parameter contribution of the silica core $\gamma_{c o}(b)$ the nonlinear parameter contribution of the cladding $\gamma_{\mathrm{cl}}$, (c) the whole effective nonlinear parameter $\gamma_{\mathrm{eff}}$, and (d) the $\gamma_{\mathrm{cl}} / \gamma_{\mathrm{eff}}$ ratio. Wavelength $\lambda=532 \mathrm{~nm}$.

core are maximum. Consequently, it is clear that $\gamma_{\mathrm{co}}$ is strongly greater for nanofiber in air than in liquids (Fig. 1a). For small diameters, the core contribution drastically decreases and tends to zero since the fundamental mode spreads out spatially with increasing mode effective area. Concerning the cladding contribution (Fig. 1b), the evolution is nearly similar. Since the intensity fraction in the evanescent field increases with decreasing nanofiber diameter, $\gamma_{\mathrm{cl}}$ increases up to reach a maximum and to decrease thereafter. This peak value corresponds to an optimal balance between the increase of the evanescent field that gives rise to the Kerr effect enhancement and to the decrease of the field intensity. For a given nanofiber diameter, the dependence of $\gamma_{\mathrm{cl}}$ on the cladding materials follows the order of their Kerr indices.

Figure 1(c) shows the effective Kerr parameter $\gamma_{\text {eff. }}$ First, it is clear that the Kerr parameter $\gamma_{\text {eff }}$ remains maximum for a nanofiber in air, even if the contribution of the evanescent field to the Kerr effect is null, and even compared to a nanofiber immersed in acetone which presents the highest Kerr coefficient $\mathrm{n}_{2}$. This is because the effective area of the fundamental mode for a nanofiber in air remains the smallest, leading to high field intensity and Kerr effect. Although $\gamma_{\text {eff }}$ is lower for a nanofiber immersed in a liquid, the contribution of the evanescent field to the Kerr effect may be comparable or even higher than that from the silica core. For acetone, $\gamma_{\text {eff }}$ has a maximal value for a core diameter of $\mathrm{d}=410 \mathrm{~nm}$, and almost $80 \%$ of the Kerr parameter is in the evanescent field. If we now compare the role of ethanol and acetone that have the same effective mode area, the contribution $\gamma_{\mathrm{co}}$ is therefore equivalent for both liquids (Fig. 1b). However, as the Kerr coefficient $\mathrm{n}_{2}$ of acetone is higher than that of ethanol, the contribution $\gamma_{\mathrm{cl}}$ is clearly greater for acetone. Thus, the effective Kerr coefficient is about 2

times larger for acetone than ethanol. The same analysis can be performed when comparing water and ethanol. A nanofiber in water will have a lower evanescent field than in ethanol due to the different step refractive index. However, water has a lower Kerr coefficient than ethanol, leading to a highest cladding contribution $\gamma_{\mathrm{cl}}$ in ethanol. These two contributions have opposite behavior and give rise to similar nonlinear parameters $\gamma_{\text {eff }}$ for both liquids (Fig. 1c). By comparing the outer fraction of field and the $\gamma_{\mathrm{cl}} / \gamma_{\mathrm{eff}}$ ratio, the contribution of the evanescent field to the Kerr effect is higher than the outer fraction of the field: the Kerr effect is not only related to the spatial extent of the electric field but also to the Kerr coefficient of the nonlinear liquid. Thus, for a nanofiber in acetone, the ratio $\gamma_{\mathrm{cl}} / \gamma_{\text {eff }}$ reaches $50 \%$ for a nanofiber diameter of $550 \mathrm{~nm}$, while the outer fraction of the field is only $39 \%$.

Our experimental approach for the observation of the evanescent Kerr effect is based on using stimulated Raman-Kerr scattering (SRKS) [4]. This inelastic light scattering comes from the inertial nature of reorientation motion of anisotropic molecules of many liquids, and gives rise to a broad asymmetric broadening of the Raman Stokes spectrum towards the red. By immersing a nanofiber in acetone, we indeed observe a large broadening of the Raman spectral components on the Stokes side, and conclude to the SRKS process, and thus the conjugated effect evanescent Raman and Kerr effects, to explain this phenomenon.

\section{Conclusion}

We theoretically and experimentally investigated the optical Kerr effect in the evanescent field of a silica nanofiber surrounded by nonlinear liquids such as water, ethanol and acetone. We have shown that the effective Kerr coefficient has two contributions: one from the silica core, which depends on the fiber diameter and follows the inverse of the effective area, the other from the cladding via the evanescent field, which depends on the diameter of the nanofiber and the nonlinear properties of the external environment

This project has received funding from the Agence Nationale de la Recherche (ANR) (ANR-16-CE24-0010; ANR-15-IDEX-0003, EIPHI graduate school ANR-17EURE-0002) and the European union H2020 program MSCA-ITN-SUPUVIR under grant agreement 722380, and the Conseil Regional de Bourgogne Franche-Comte.

\section{References}

1. L. Tong and M. Sumetsky, Springer, 978-3-64203362 (2011).

2. L. Shan, G. Pauliat, G. Vienne, L. Tong, and S. Lebrun, Appl. Phys. Letters 102, 201110 (2013)

3. S. Afshar V., and T. M. Monro, Opt. Express 17(4), 2298-2318 (2009)

4. G. S. He, R. Burzynski, and P. N. Prasad, J. Chem. Phys. 93, 7647-7655 (1990) 